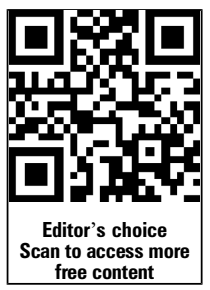

- Additional material is published online only. To view please visit the journal online (http://dx.doi.org/10.1136/ gutjnl-2013-306520)

For numbered affiliations see end of article.

\section{Correspondence to} Dr Sebastian Zeissig, Department of Internal Medicine I, University Medical Center Schleswig-Holstein,

Kiel, Schittenhelmstr. 12, Kiel 24105, Germany: szeissig@1med.uni-kiel.de and Dr Andre Franke, Institute of Clinical Molecular Biology, Christian-Albrechts-University of Kiel, Schittenhelmstr. 12 24105 Kiel, Germany; a.franke@mucosa.de

YZ and BSP contributed equally to this work. SZ and $\mathrm{AF}$ contributed equally to this work.

Received 2 December 2013 Revised 6 January 2014 Accepted 30 January 2014 Published Online First 26 February 2014

\title{
XIAP variants in male Crohn's disease
}

\author{
Yvonne Zeissig, ${ }^{1,2,3}$ Britt-Sabina Petersen, ${ }^{3}$ Snezana Milutinovic, ${ }^{4}$ Esther Bosse, ${ }^{1}$ \\ Gabriele Mayr, ${ }^{3}$ Kenneth Peuker, ${ }^{1}$ Jelka Hartwig, ${ }^{1}$ Andreas Keller, ${ }^{5}$ Martina Kohl, ${ }^{2}$ \\ Martin W Laass, ${ }^{6}$ Susanne Billmann-Born, ${ }^{3}$ Heide Brandau, ${ }^{7}$ Alfred C Feller, ${ }^{8}$ \\ Christoph Röcken, ${ }^{9}$ Martin Schrappe, ${ }_{1}^{2}$ Philip Rosenstiel, ${ }^{3}$ John C Reed, ${ }_{1}^{4}$ \\ Stefan Schreiber, ${ }^{1,3}$ Andre Franke, ${ }^{3}$ Sebastian Zeissig ${ }^{1}$
}

\section{ABSTRACT \\ Objective The genetic basis of inflammatory bowel} disease (IBD) is incompletely understood. The aim of this study was to identify rare genetic variants involved in the pathogenesis of IBD.

Design Exome sequencing and immunological profiling were performed in a patient with early onset Crohn's disease (CD). The coding region of the gene encoding $X$-linked inhibitor of apoptosis protein (XIAP) was sequenced in samples of 275 paediatric IBD and 1047 adult-onset CD patients. XIAP genotyping was performed in samples of 2680 IBD patients and 2864 healthy controls. Functional effects of the variants identified were investigated in primary cells and cultured cell lines.

Results Our results demonstrate the frequent occurrence of private variants in XIAP in about four percent of male patients with paediatric-onset $C D$. While $X I A P$ mutations are known to be associated with the primary immunodeficiency (PID) X-linked

lymphoproliferative disease type 2 (XLP2), CD patients described here exhibited intestinal inflammation in the absence of XLP2 and harboured a spectrum of mutations partially distinct from that observed in XLP2. The majority of XIAP variants identified was associated with a selective defect in NOD1/2 signalling, impaired NOD1/2-mediated activation of NF- $\mathrm{KB}$, and altered NF$\kappa \mathrm{B}$-dependent cytokine production.

Conclusions This study reveals the unanticipated, frequent occurrence of XIAP variants in male paediatriconset CD. The link between XIAP and NOD1/2, and the association of XIAP variants with $X L P 2$, support the concept of PID in a subset of IBD patients. Moreover, these studies provide a rationale for the implementation of XIAP sequencing in clinical diagnostics in male patients with severe $C D$.

\section{INTRODUCTION}

Inflammatory bowel disease (IBD) is a heterogeneous group of chronic inflammatory disorders of the intestine with Crohn's disease (CD) and ulcerative colitis (UC) as the two major forms. ${ }^{1}$ Although the precise aetiology of IBD is unknown, susceptibility to disease is influenced by a complex interplay of genetic and environmental factors. ${ }^{2-4}$ Previously, we and others identified over 163 common genetic variants associated with IBD that are involved in the regulation of intestinal barrier function as well as innate and adaptive immunity. ${ }^{5}$ However, for the majority of genetic variants associated with IBD, a

\section{Significance of this study}

What is already known on this subject?

- Immune defects have been described in patients with $C D$ and may contribute to the pathogenesis of intestinal inflammation.

- XIAP mutations are associated with X-linked lymphoproliferative disease type 2 (XLP2), a primary immunodeficiency, which can manifest as intestinal inflammation.

- XIAP has been implicated in the regulation of several pathways relevant to IBD including NOD1/2 signalling, apoptosis, and natural killer $\mathrm{T}$ cell development.

\section{What are the new findings?}

- Private variants in XIAP are commonly observed in male patients with paediatric-onset $C D$ and affect about $4 \%$ of patients in this subset of CD patients in the German cohort described here.

- Novel XIAP variants described here are associated with $C D$ as the sole clinical manifestation in the absence of XLP2.

- Lack of functional XIAP in primary immune cells is associated with selective defects in NOD1/2 signalling, while other alterations in innate and adaptive immunity were not observed.

\section{How might it impact on clinical practice in} the foreseeable future?

- These studies provide a mechanistic basis for the unexplained observation of functional NOD2 defects in the absence of genetic NOD2 variants, support the concept of immunodeficiency in a subset of CD patients, and provide a rationale for the implementation of XIAP sequencing in routine clinical diagnostics in male patients with severe CD.

complete understanding of the mechanisms that contribute to disease pathogenesis is missing. This is related to the fact that these polymorphisms are common, of low to moderate effect size, and are likely to modify disease risk through interaction with environmental factors and other genetic variants found in the same individual. ${ }^{36}$ 
Mendelian disorders can provide crucial insight into the mechanisms of disease pathogenesis. Accordingly, the analysis of gene-function relationships in IBD may be facilitated by the study of rare phenotypes such as early onset CD and UC, which are often associated with a severe course of disease, lack of response to immunomodulators and biologicals, and familial segregation. $^{7-12}$ In accordance with this concept, studies in early onset IBD revealed apparent monogenetic variants of IBD and provided unique insight into disease pathogenesis. Thus, mutations in the genes encoding for IL-10 and its receptor subunits IL-10R1 and IL-10R2 were found to be associated with chronic intestinal inflammation and manifestation of disease during the first year of life..$^{9-11} 1314$ As Il10 and Il10rb deficiency in mice are associated with spontaneous intestinal inflammation, these data suggest that defects in this pathway are sufficient to induce intestinal inflammation in the presence of the intestinal microbiota. ${ }^{15} 16$ Similarly, mutations in a disintegrin and metalloprotease 17 (ADAM17) are associated with early onset IBD in accordance with susceptibility to intestinal inflammation observed in mice with Adam 17 deletion. ${ }^{17} 18$ Further, the study of primary immunodeficiencies (PID), such as chronic granulomatous disease (CGD), Wiskott-Aldrich syndrome, and IPEX has revealed monogenetic immune defects associated with chronic intestinal inflammation. ${ }^{8} 1920$ These studies demonstrate how individual genetic variants can lead to intestinal inflammation and, together with functional studies in CD patients, ${ }^{21}{ }^{22}$ lend support to the concept of primary immune defects underlying a subset of IBD cases. ${ }^{8} 2324$ Along these lines, recent studies revealed that mutations in the gene encoding for the $\mathrm{X}$-linked inhibitor of apoptosis protein (XIAP) are associated with the PID XLP2 and also with chronic intestinal inflammation. ${ }^{25-30}$ While these observations were originally made in families with XLP2, a recent exome sequencing study identified a novel XIAP variant in a child with early onset CD as the sole clinical manifestation. ${ }^{31}$ These results raised the question of whether XIAP variants are commonly associated with IBD. Here, we demonstrate the frequent occurrence of private $X I A P$ variants in male patients with paediatric-onset $C D$, which affects about $4 \%$ of patients in this CD subset.

\section{METHODS}

\section{Patients}

The study was approved by the local ethics committees. All patients and, in case of children, both parents provided written informed consent. Control subjects for functional studies included the patient's mother and healthy volunteers (median age 25 years, range 23-33). Neither the patient nor the controls received medication. Genotyping of XIAP variants was performed in $1900 \mathrm{CD}$ patients (median age at disease onset 25 years, range $1-73 ; 58 \%$ male, $42 \%$ female), 780 UC patients (median age at disease onset 27, range 3-61; 54\% male, 46\% female) and 2864 healthy controls (52\% male, $48 \%$ female). Sanger sequencing of XIAP was performed in two cohorts: A cohort of 275 paediatric IBD cases including 181 CD cases (95 male patients, 86 female patients, median age at disease onset 13, range $1-16 ; 37$ cases $\mathrm{A} 1 \mathrm{a}, 144$ cases $\mathrm{A} 1 \mathrm{~b}$ according to the Paris classification ${ }^{12}$ ) and 94 UC cases (48 male patients, 46 female patients, median age at disease onset 13 years, range 1-16; 24 cases A1a, 70 cases A1b according to the Paris classification $^{12}$ ). The second cohort consisted of 1047 patients with adult onset $\mathrm{CD}$ (median age at disease onset 29 years, range 17-70, 36\% male, 64\% female).

\section{Exome sequencing}

Samples were enriched using Illumina's TruSeq Exome Enrichment Kit. Sequencing of $2 \times 100$ bp paired-end reads was performed for the three samples together on one lane of the Illumina HiSeq2000. Reads were mapped against the human reference genome build hg19 using BWA ${ }^{32}$ v0.5.9, sorted, converted to bam format and indexed with SAMtools ${ }^{33} \mathrm{v} 0.1 .8$, followed by the removal of PCR duplicates with Picard v1.55 (http://picard.sourceforge.net). Local realignment around InDels and base quality score recalibration as well as variant calling and quality score recalibration were performed with GATK $^{34}$ v1.260 according to their best practice recommendations. For annotation we applied ANNOVAR's ${ }^{35}$ summarize_annovar.pl script. For the identification of de novo variants we used a two-step approach involving the somatic mutation command from Varscan $^{36} \mathrm{v}$ 2.2.11 for a pairwise comparison of the son and the mother as well as the son and the father. We calculated the overlap of detected differences from both comparisons to find variants present exclusively in the son followed by manual inspection of the alignment at positions of potential de novo mutations.

\section{Sanger sequencing}

PCR and Sanger sequencing were performed on an Applied Biosystems 3730xl. Sequences were analysed using novoSNP. ${ }^{37}$ Potential novel coding variants were validated by resequencing in the forward and reverse directions. The average overall drop-out rate was below 2.3\%. PCR primers and conditions are shown in online supplementary table S1.

\section{Protein structure analysis}

See online supplementary methods.

\section{Flow cytometry}

Flow cytometry was performed as described previously ${ }^{38}$ and as described in online supplementary methods.

\section{T cell proliferation and apoptosis}

For restimulation-induced cell death, peripheral blood mononuclear cells (PBMCs) were stimulated with PHA $(2 \mu \mathrm{g} / \mathrm{mL})$. After $24 \mathrm{~h}$, recombinant hIL-2 (100 U/mL, R\&D systems) was added and renewed every $48 \mathrm{~h}$. On day 14, cells were restimulated with $1 \mu \mathrm{g} / \mathrm{mL}$ plate-bound anti-CD3 antibody (OKT3, Biolegend). Apoptosis was determined using the FITC Annexin $\mathrm{V}$ kit (BD Biosciences).

$\mathrm{T}$ cell proliferation was determined by ${ }^{3} \mathrm{H}$-thymidine incorporation 5 days after stimulation (PHA or anti-CD3) or restimulation (PHA, followed by plate-bound anti-CD3 after 14 days) as described previously. ${ }^{38}$ 5-bromo-2'-deoxyuridine (BrdU) incorporation and cell cycle analysis were determined using the FITC BrdU Flow Kit (BD Biosciences).

\section{TLR and NOD signalling}

$1 \times 10^{5}$ PBMCs were stimulated in 96-well flat-bottom plates with LPS (Sigma-Aldrich), MDP (InvivoGen), Tri-DAP (Invivogen), Pam3CSK4 (InvivoGen), TNF $\alpha$ (Peprotech), PMA (Sigma-Aldrich), and ionomycin (Sigma-Aldrich). Cytokine secretion was detected by ELISA (R\&D) $24 \mathrm{~h}$ after stimulation. Where indicated, Z-VAD-fmk or Z-YVAD-fmk (Enzo Life Sciences) was added.

For luciferase assays, an NF-кB-dependent firefly luciferase plasmid (Promega) was transfected (Lipofectamine 2000) along with wildtype or mutant XIAP and a Renilla luciferase plasmid 
(phRluc, Promega) for normalisation. After $24 \mathrm{~h}$, cells were stimulated as indicated, and luciferase expression was analysed after another 16 h (Dual-Glo-Luciferase Assay System, Promega).

Electrophoretic mobility shift assays were performed as described previously ${ }^{39}$ using a probe, which contains an NF-kB consensus site $\left(5^{\prime}\right.$-AGT TGA GGG GAC TTT CCC AGG C-3').

\section{Western blotting and immunoprecipitation}

Immunoprecipitation and western blotting was performed as described previously, ${ }^{40}$ and as described in online supplementary methods.

\section{Monocyte-derived dendritic cells (DCs) and lentiviral reconstitution}

Generation of monocyte-derived DCs and lentiviral infection were performed as described previously ${ }^{38}$ and as outlined in online supplementary methods.

\section{Statistical analysis}

Statistical testing was performed using the unpaired Student $t$ test. $p$ Values were calculated in a two-tailed manner. For multiple comparisons against the same control, one-way analysis of variance (ANOVA) with Dunnett's posthoc test was applied.

\section{RESULTS}

\section{A novel nonsense mutation in XIAP revealed by exome sequencing}

A male patient presented with bloody diarrhoea at the age of 9 months. Endoscopy and histological examination revealed severe, ulcerative, discontinuous pancolitis with anal fissures and stenosis. Low-grade, chronic, Helicobacter pylori-negative gastritis and duodenitis in accordance with upper gastrointestinal CD was demonstrated. The patient was classified as A1a, L2+L4a, $\mathrm{B} 2 \mathrm{p}$ according to the Paris classification ${ }^{12}$ and the subsequent course of disease was refractory to mesalazine, corticosteroids, antibiotics and infliximab. A loop ileostomy at 36 months of age was associated with clinical improvement despite persistent mild, discontinuous colitis. For further clinical characterisation see the Supplementary Results section, online supplementary tables S2 and S3, and. ${ }^{30}$ The patient and both parents underwent exome sequencing. The average sequencing coverage ranged between 45 -fold and 80 -fold with over $90 \%$ of the target covered by more than eightfold in all samples (online supplementary table S4). We identified a total of 53320 on-target variants in the patient with 22452 coding variants of which 1212 nonsynonymous variants (missense, nonsense, cancel-start, readthrough, splice-site and frameshift) were not present in dbSNP132. We filtered using a maximum frequency of $1 \%$ in the 1000 genomes data and inspected the remaining 995 variants assuming a recessive model with homozygosity or compound heterozygosity in the son and heterozygosity in both parents. This approach yielded one homozygous (FAM151A) and two compound-heterozygous (RAPGEF4, DCHS2) variants (online supplementary table S5). Neither RAPGEF4 nor DCHS2 are expressed in the intestine. Expression of DCHS2, encoding dachsous cadherin-related 2, is restricted to brain and testis, ${ }^{41}$ while expression of RAPGEF4, encoding Rap guanine nucleotide exchange factor 4 , is restricted to the central nervous system, adrenal gland and pancreas. ${ }^{42} 43$ Additionally, neither the function nor the distribution of FAM151A is known. Given that these variants did not provide sufficient mechanistic explanation for the observed clinical phenotype, we investigated the possibility of a de novo mutation in the affected son. This resulted in the identification of a novel, hemizygous, de novo nonsense mutation in XIAP (c.295G > T, E99X (p.Glu99*), table 1, figure 1A), while other de novo mutations were not detectable. The affected residue is located in the second of seven exons and truncates the protein after the first of three baculovirus inhibitor of apoptosis protein repeat (BIR) domains (figure 1A). This is associated with loss of function specific to BIR domains 2 and 3 and the RING domain, but predicted to retain the ability of the BIR1 domain to interact with TAB1 (figure 1B).

\section{Unaltered PBMC subsets and T cell function in the absence of functional XIAP}

To investigate the mechanistic basis underlying intestinal inflammation in the presence of XIAP variants, we studied the phenotype and function of immune cells in the patient harbouring XIAP E99X. Full-length XIAP was undetectable in various PBMC subsets (figure 2A) and lymphoblastic B cell lines (figure 2B). In accordance with a de novo mutation, PBMCs of the patient's mother exhibited unaltered XIAP expression (figure 2A). Phenotypic PBMC analyses revealed unaltered absolute and relative numbers of monocytes, $\mathrm{T}$ cells and $\mathrm{B}$ cells in the patient carrying XIAP E99X (figure 2C-D, see online supplementary table S3, and data not shown). CD4 and CD ${ }^{+} \mathrm{T}$ cells in the affected child contained a higher proportion of naive subsets compared with healthy adults (figure 2E, see online supplementary figure S1), but the distribution of naive and memory $\mathrm{T}$ cells reflected that previously found in healthy children. ${ }^{44}$ Similarly, reduced numbers of memory but not total B cells and unaltered numbers of peripheral blood plasma cells and immunoglobulin levels were found (figure 2C, F, see online supplementary table S3). While deficiency in invariant natural killer T (iNKT) cells was described in patients with XIAP variants, ${ }^{25}$ the patient carrying XIAP E99X exhibited detectable levels of iNKT cells at the lower margin of controls (figure $2 \mathrm{G}$ and reference ${ }^{38}$ ).

XIAP BIR domains inhibit caspase-3 (BIR2), -7 (BIR2), and -9 (BIR3). ${ }^{28}$ Accordingly, XIAP deficiency was reported to be associated with increased susceptibility to reactivation-induced T cell death (RICD). ${ }^{25}{ }^{45}$ However, despite a mutation causing deletion of the BIR2 and BIR3 domains of XIAP, CD4+ and $\mathrm{CD}^{+} \mathrm{T}$ cells from the XIAP E99X patient did not exhibit alterations in activation-induced or restimulation-induced $\mathrm{T}$ cell death (figure 3A and data not shown). Further, T cell proliferation (figure 3B), expansion (figure 3C), and cell cycle

Table 1 Summary of identified XIAP variants

\begin{tabular}{llllllll}
\hline & Nucleotide sequence & Exon location & Protein sequence & Gender & Zygosity & IBD type & Age at disease onset (years) \\
\hline Patient 1 & c.295G $>$ T & 2 & E99X & Male & Hemizygous & CD & 0.75 \\
Patient 2 & C.115G $>$ T & 2 & G39C & Male & Hemizygous & CD & 13 \\
Patient 3 & c.890A $>$ C & 3 & K297T & Male & Hemizygous & CD & 15 \\
Patient 4 & c.968G $>$ A & 3 & W323X & Male & Hemizygous & CD & 16 \\
\hline
\end{tabular}


A


Figure 1 Summary of XIAP variants. A: Domain architecture of the human XIAP protein. Missense mutations are indicated in grey, and nonsense mutations in red boxes. E99X truncates the XIAP protein immediately after the BIR1 domain (residues 26-93), while W323X truncates XIAP near the C-terminus of BIR3 (residues 265-330). B: BIR1/TAB1 complex with XIAP E99X and G39C. Cartoon and surface representation of the XIAP BIR1 protein domain (green) in complex with TAB1 (yellow). The location of E99X is indicated by a red line, and the deleted C-terminal segment is coloured in red. The truncation likely retains the BIR1 domain unaffected with respect to domain interaction and fold stability. G39C is represented as spheres in atomic colours.

progression (figure 3D) induced by phytohemagglutinin (PHA) and anti-CD3 treatment were unaltered (figure 3B-D and data not shown).

\section{XIAP E99X is associated with a severe and selective defect in NOD signalling}

XIAP was shown to be critical for NF- $\mathrm{BB}$-dependent production of IL-6 and IL-8 in response to NOD2 signalling. ${ }^{31} 40 \quad 46-49$ In accordance with these findings, PBMCs from the patient carrying XIAP E99X did not exhibit IL-6 or IL-8 secretion in response to the NOD2 ligand muramyl dipeptide (MDP) (figure 4A and see online supplementary figure S2A). Exome sequencing results demonstrated that impaired NOD2 signalling was not due to coding variants in NOD2, BIRC2, BIRC3, FRMPD2, RBCK1, RIPK2, RNF31, SHARPIN and TRAF2, all of which are associated with NOD2 and contribute to NOD2 signalling (data not shown). ${ }^{40}$ 47-49 LPS-induced, Pam3CSK4-induced and TNF $\alpha$-induced IL- 6 and IL- 8 secretion were unimpaired, thus confirming the absence of general defects in toll-like receptor (TLR) signalling or NF- $\mathrm{BB}$-mediated IL-6 and IL-8 secretion (figure 4B$\mathrm{D}$, see online supplementary figure S2B, and data not shown). Additionally, while XIAP has been suggested to act as a negative regulator of the NLRP3 inflammasome, ${ }^{50}$ caspase- 1 cleavage and caspase-1-mediated IL-1 $\beta$ secretion were unaltered in the patient carrying XIAP E99X (see online supplementary figure S3A-B).

XIAP binds receptor-interacting serine-threonine kinase 2 (RIPK2), a downstream mediator of NOD2, in a manner dependent on its BIR2 domain. ${ }^{40} 49$ Since XIAP E99X lacks the
BIR2 domain, we investigated whether the truncated protein fails to interact with RIPK2. While wildtype and E99X myc-XIAP were expressed at similar levels, RIPK2 co-immunoprecipitated only with wildtype but not E99X XIAP in $293 \mathrm{~T}$ cells (figure 4E). XIAP E99X thus fails to bind to RIPK2, which provides a mechanistic explanation for the observed NOD2 defect.

To confirm that impaired NOD2 signalling is the consequence of altered XIAP function, XIAP expression was restored by lentiviral reconstitution in primary monocyte-derived dendritic cells (moDCs) of the XIAP E99X patient. Viral transduction rates as determined by lentivirus-derived green fluorescent protein (GFP) were similar in all patients with about $70 \% \mathrm{GFP}^{+}$cells among transduced moDCs (see online supplementary figure S4) in accordance with previous results. ${ }^{38}$ Importantly, XIAP transduction restored baseline and MDP-induced IL-8 secretion in XIAP E99X moDCs (figure 4F). By contrast, moDCs from healthy controls did not exhibit increased baseline or MDP-induced IL-8 secretion upon lentiviral XIAP transduction, thus demonstrating that XIAP expression is not rate-limiting for NOD2 signalling in moDCs from healthy controls (figure 4F). Together, these results demonstrate that impaired NOD2 signalling is the consequence of the XIAP E99X variant.

\section{Targeted sequencing reveals additional CD-associated variants in XIAP}

Another case of early onset $C D$ associated with a hemizygous missense mutation (C203Y) in XIAP has recently been 
A



C



$\mathrm{E}$

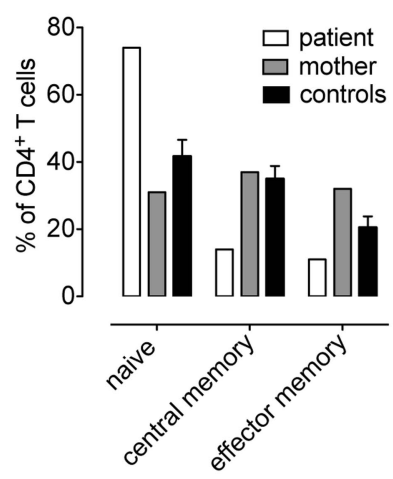

$\mathrm{CD}^{+} \mathrm{T}$ cells $\mathrm{CD}^{+} \mathrm{T}$ cells $\quad \mathrm{CD}^{+} \mathrm{T}$ cells

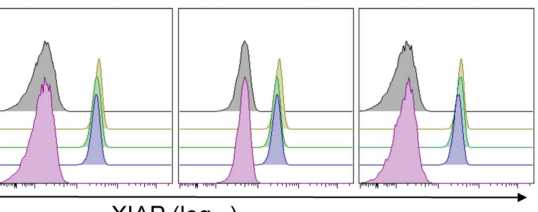

XIAP $\left(\log _{10}\right)$
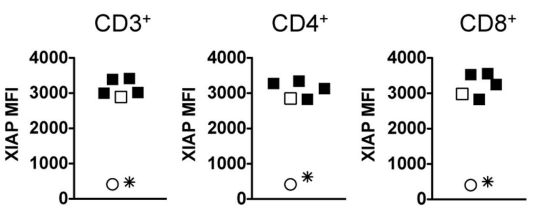

B

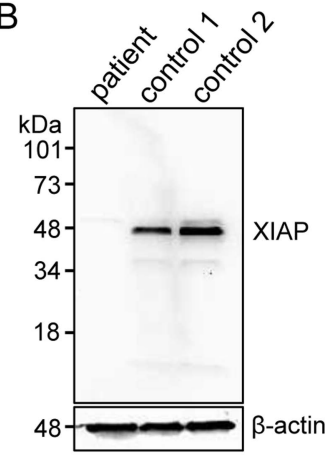

$\mathrm{F}$

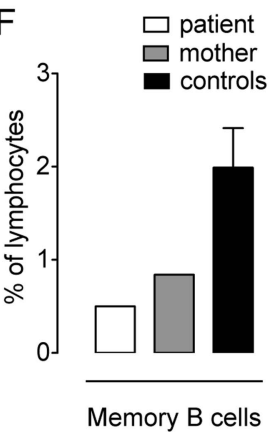

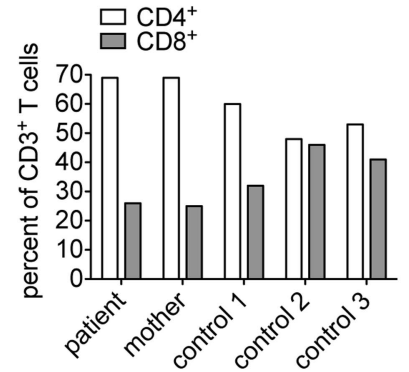

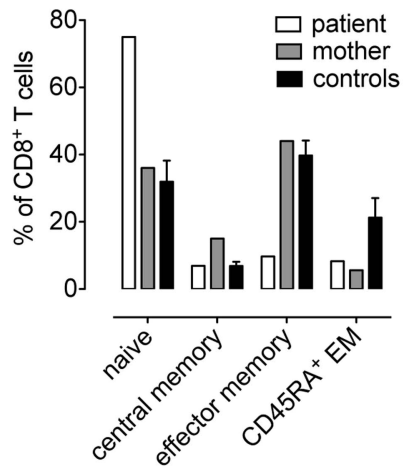



Figure 2 The XIAP E99X variant is not associated with alterations in PBMC frequencies. A: XIAP expression of the indicated PBMC subsets as measured by intracellular flow cytometry. Histograms are shown in the upper panel and mean fluorescence intensities (MFI) are given in the lower panel. B: XIAP protein expression as determined by western blot analysis in lymphoblastic cell lines. C-F: characterisation of PBMC subsets by flow cytometry. C: percentage of CD14 $4^{+}$monocytes, $C D 19^{+} B$ cells, $C D 3^{+} T$ cells among PBMCs. D: percentage of $C D 4$ and $C D 8^{+}$among $C D 3^{+} T$ cells.

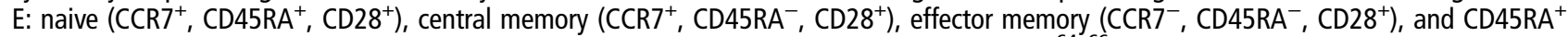
effector memory $\left(\mathrm{CCRR}^{-}, \mathrm{CD} 45 \mathrm{RA}^{+}, \mathrm{CD} 28^{+}\right)$subsets of $\mathrm{CD} 4$ and $\mathrm{CD} 8^{+} \mathrm{T}$ cells according to ref ${ }^{64-66}$. F: percentage of $\mathrm{CD} 19^{+}, \mathrm{CD}^{2} 7^{+}, \mathrm{CD} 38^{-}$ peripheral memory B cells and $\mathrm{CD} 19^{+}, \mathrm{CD} 27^{+}, \mathrm{CD} 38^{+}$plasma cells. F: percentage of $\alpha$-galactosylceramide (PBS57)/CD1d-tetramer-positive $\mathrm{CD}^{+}$ iNKT cells. In C, E, F, mean \pm sem of three healthy controls are shown. In all other panels, individual controls are shown. Statistical analysis is not provided, as studies with primary PBMCs from the XIAP E99X patient were performed only once.

identified. ${ }^{31}$ To test for potential recurrence of XIAP variants, we carried out genotyping of the E99X and C203Y mutations in $1900 \mathrm{CD}$ patients, 780 UC patients and 2864 healthy controls (see Methods). Genotyping results were monomorphic (data not shown), suggesting a private character of these two variants. 
Figure 3 Unaltered apoptosis and proliferation of $T$ cells obtained from the XIAP E99X patient. A: PBMCs were stimulated with phytohemagglutinin (PHA) in the presence of IL-2.

Reactivation was induced by anti-CD3 cross-linking. Reactivation-induced cell death was determined by Annexin V and 7-AAD staining. $B$ :

activation-induced $\mathrm{T}$ cell proliferation induced by PHA and measured by ${ }^{3} \mathrm{H}$-thymidine incorporation in total $\mathrm{T}$ cells. C: fold expansion of the indicated $T$ cell subsets 14 days after PHA treatment. D: cell cycle analysis of the indicated $T$ cell subsets two days after anti-CD3 cross-linking as determined by flow cytometric BrdU/ 7-AAD analysis. CD4 T cells are shown in the upper panel. CD8 ${ }^{+} \mathrm{T}$ cells are shown in the lower panel. In B, mean \pm sem of triplicate cultures is shown. In all other panels, individual controls are shown. Statistical analysis is not provided as studies with primary PBMCs from the XIAP E99X patient were performed only once.
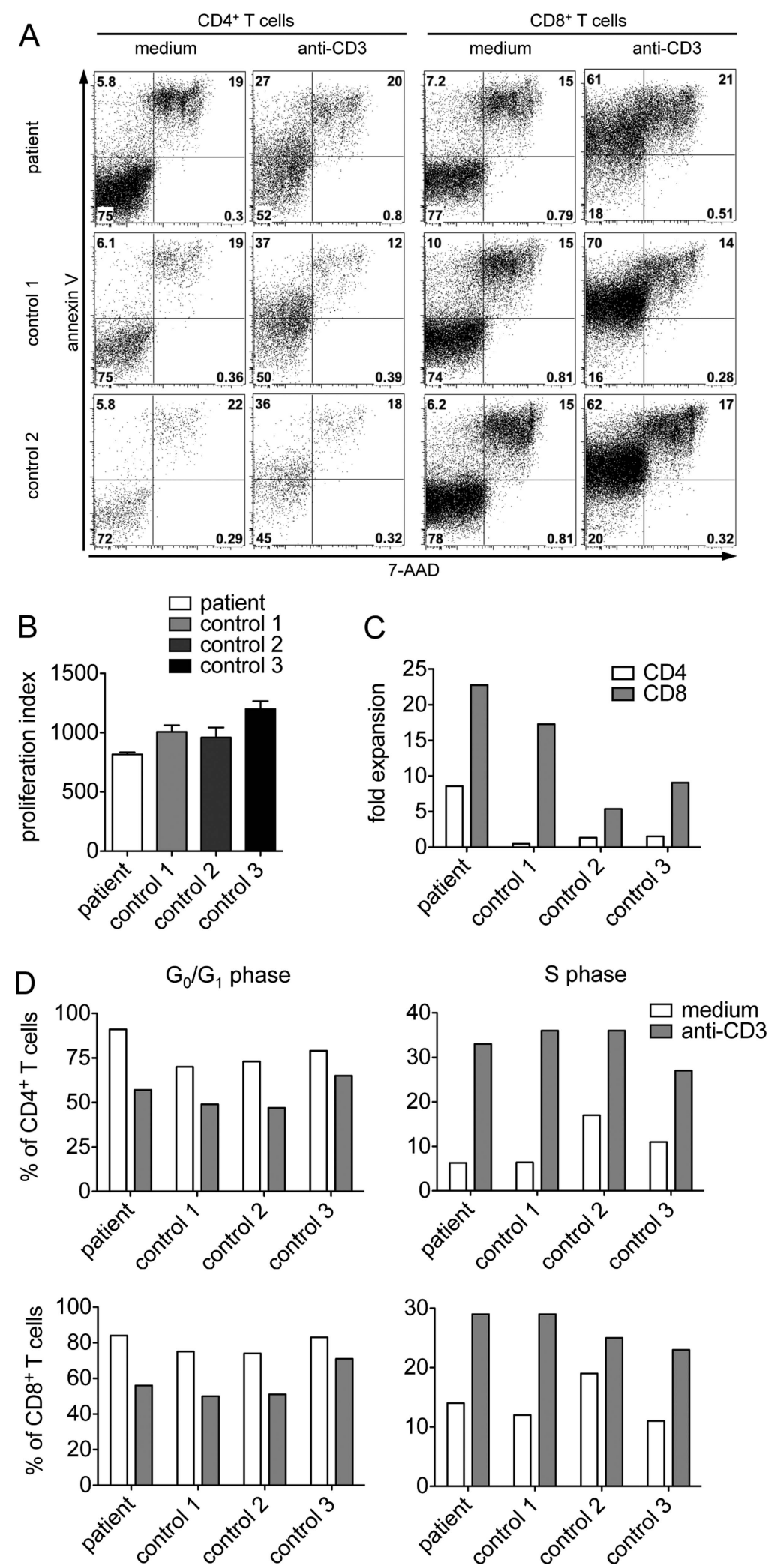
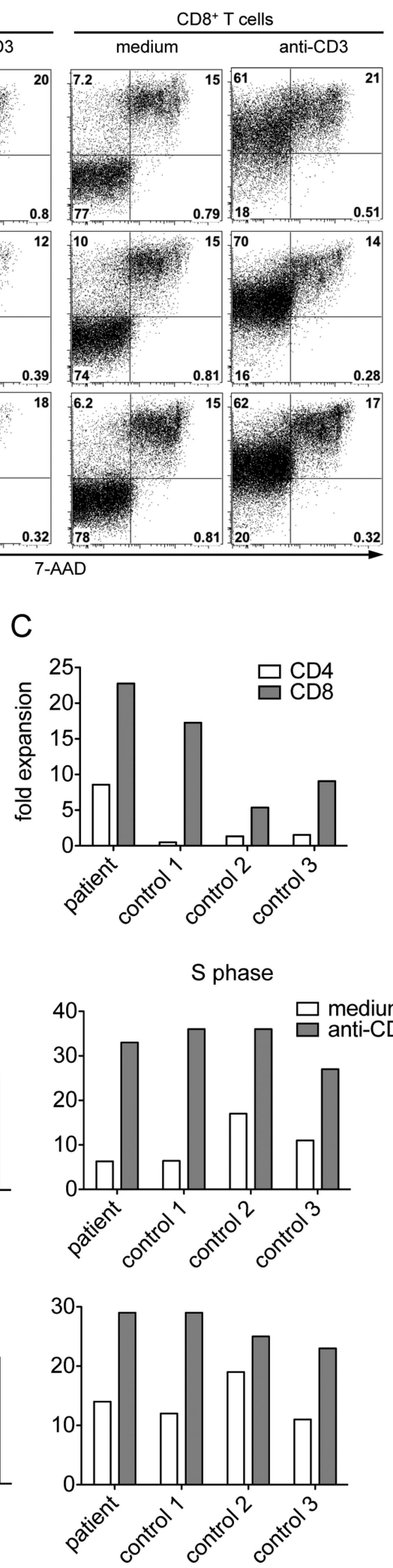

To investigate whether additional XIAP variants can be identified in patients with IBD, targeted Sanger sequencing was performed in a cohort of 275 paediatric-onset IBD cases (age $\leq 16$ years according to groups A1a and A1b of the Paris classification, ${ }^{12}$ ) and 1047 adult-onset CD patients ( $\geq 17$ years according to groups A2 and A3 of the Montreal classification; ${ }^{51}$ see Methods for further details). Sanger sequencing revealed four novel variants in XIAP including one synonymous, two missense and one nonsense variant (table 1 ). Non-synonymous mutations occurred exclusively in males with paediatric-onset CD (see Suppl. Results), were hemizygous, and located in exons 2 and 3 of the XIAP gene (table 1, figure 1A). Remarkably, missense variants G39C (p.Gly39Cys) and K297T (p.Lys297Thr) were located in the BIR1 and BIR3 domains of XIAP, respectively, and 
Figure 4 A severe NOD2 defect in the XIAP E99X patient, impaired interaction of XIAP E99X with RIPK2, and functional NOD2 restoration upon lentiviral XIAP reconstitution. A-D: IL-8 secretion as determined by ELISA in supernatants of PBMCs treated with the indicated stimulants for $24 \mathrm{~h}$ (Pam3CSK4 $10 \mu \mathrm{g} / \mathrm{mL}$, TNF $\alpha 5 \mathrm{ng} / \mathrm{mL}$ ). $\mathrm{E}:$ the left panel shows coimmunoprecipitation of FLAG-tagged RIPK2 with WT but not mutant myc-tagged XIAP as determined by western blotting in $293 \mathrm{~T}$ cells. The right panel shows expression of RIPK2 and XIAP in cell lysates. F: restoration of NOD2 signalling by lentiviral reconstitution with XIAP wildtype protein in monocyte-derived dendritic cells of the XIAP E99X patient. In A-D, $F$, mean \pm sem of triplicate cultures are shown. Results in $\mathrm{E}$ are representative of two independent experiments. Studies in $A-D$ and $F$ involving primary PBMCs of the XIAP E99X patient were performed only once and statistical analysis is therefore not provided.
A

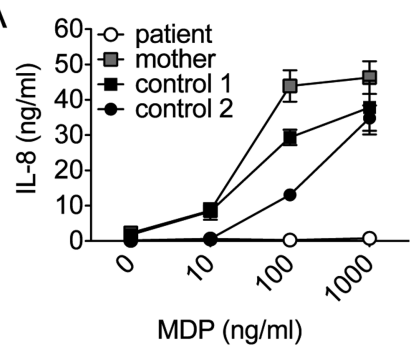

C

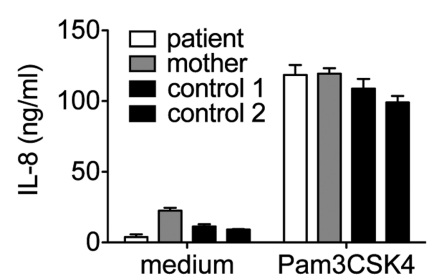

$E$
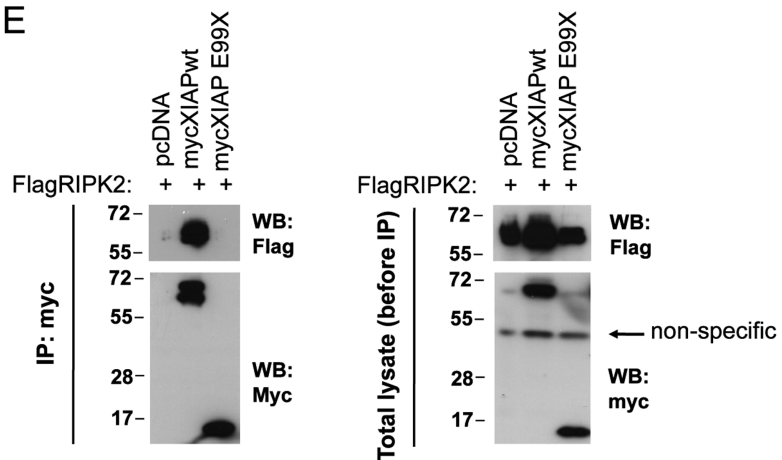

F

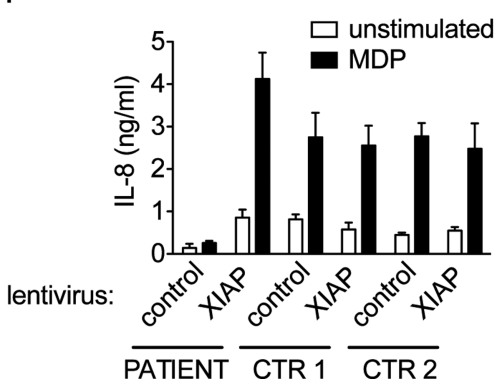

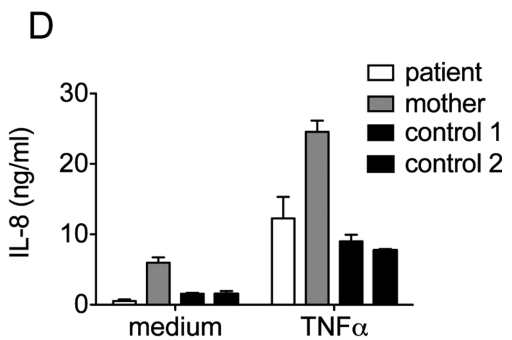

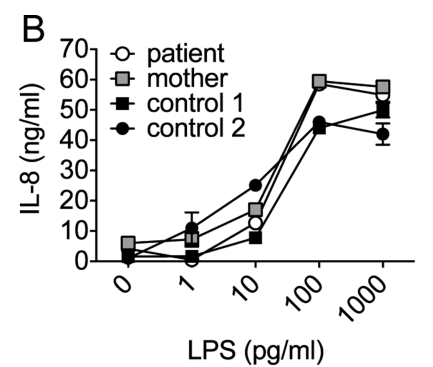
received continuous corticosteroid treatment between the ages of 16 and 52 years. Azathioprine and infliximab treatment were initiated at 50 and 53 years of age, respectively, and were discontinued due to severe respiratory infections. The patient died at age 54 years from hospital-acquired pneumonia (see Suppl. Results for further clinical details). The patient carrying XIAP K297T showed gastric, jejunal, ileal and colonic involvement of $\mathrm{CD}$ including pyloric stenosis and perianal disease. $\mathrm{CD}$ manifested at age 15 years (A1b, L3+L4a/L4b, B2 $\mathrm{p}^{12}$ ), and required combined treatment with steroids, azathioprine and infliximab for disease control (see Suppl. Results and see online supplementary figure S5 for further clinical details). Both patients were repeatedly tested negative for EBV and did not exhibit signs of haemophagocytic lymphohistiocytosis (HLH). The patient carrying XIAP G39C is a male CD patient with age of disease onset at 13 years who was enrolled at the age of 38 years in an infliximab study. Further clinical information was not available on this patient. Thus, all patients with clinical data available shared the presence of small and large intestinal involvement, perianal disease and stricturing behaviour, while the location of small intestinal disease and the presence or absence of penetrating behaviour differed among patients. 
Figure 5 Impaired NOD1/2-mediated NF- $\kappa B$-dependent signalling by XIAP E99X, W323X, and K297T. A: coimmunoprecipitation of FLAG-tagged RIPK2 with WT and mutant myc-tagged XIAP after transfection into 293 T cells. B: IL-8 secretion by XIAP-deficient HCT116 cells transfected with WT or mutant XIAP as well as the empty vector control ( $p c D N A)$ and stimulated with the indicated concentration of MDP. C: DNA binding activity of NF- $\mathrm{KB}$ as determined by electrophoretic mobility shift assay in XIAP-deficient HCT116 cells transfected with WT or mutant XIAP and stimulated with MDP $(10 \mu \mathrm{g} / \mathrm{mL})$. D: $\mathrm{NF}-\kappa \mathrm{B}$-dependent transcription as determined by expression of an $\mathrm{NF}$ - $\mathrm{B}$-dependent firefly luciferase construct after transfection into XIAP-deficient HCT116 cells along with a renilla luciferase plasmid for normalisation and WT or mutant XIAP. Cells were stimulated as indicated (MDP, Tri-DAP: $10 \mu \mathrm{g} / \mathrm{mL}$ ). E: IL-8 secretion by XIAP-deficient HCT116 cells transfected with WT or mutant XIAP or empty vector control (pcDNA) and stimulated with the indicated concentrations of TriDAP. F: IL-8 secretion by XIAP-deficient HCT116 cells transfected with WT or mutant XIAP and stimulated with LPS $(1 \mu \mathrm{g} /$ $\mathrm{mL}$ ) or PMA (50 $\mathrm{ng} / \mathrm{mL})$ and ionomycin $(1 \mu \mathrm{m})$. Mean \pm sem is shown in $B, D, E$ and $\mathrm{F}$. Results are representative of three independent experiments.
A



B

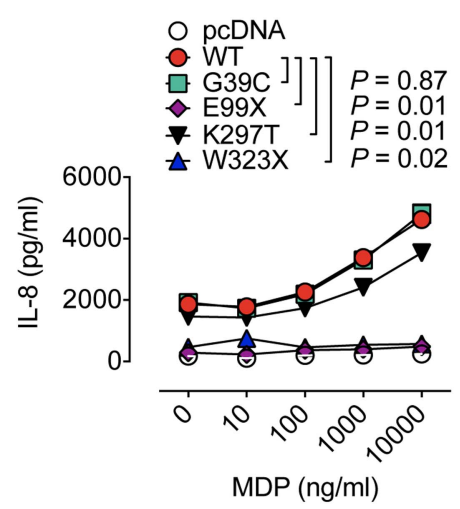

C
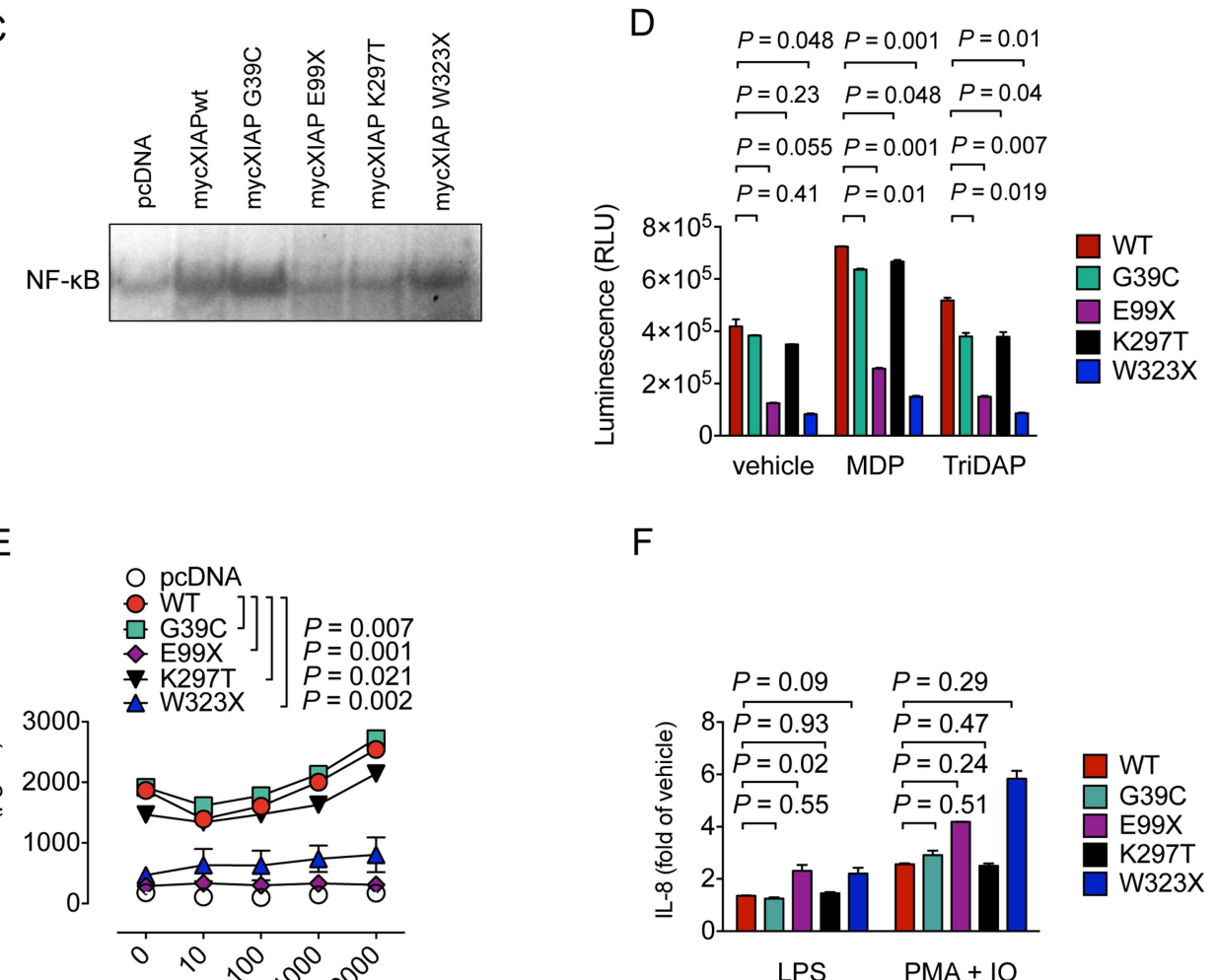

$E$

$\operatorname{TriDAP}(\mathrm{ng} / \mathrm{ml})$

$\mathrm{F}$

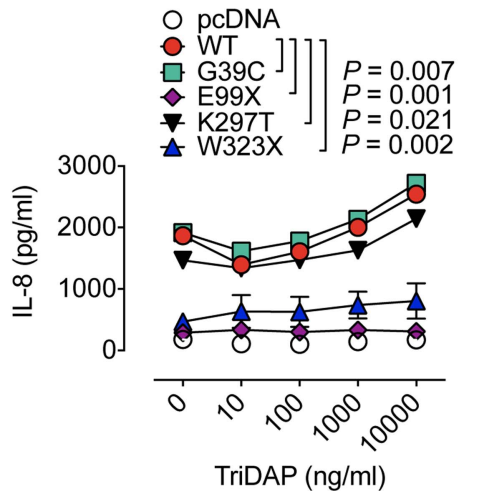

\section{XIAP W323X affects NOD1/NOD2 signalling through truncation of the RING domain}

Of the three additional XIAP variants identified, W323X is associated with loss of the XIAP RING domain, but predicted to preserve the BIR2 domain (figure $1 \mathrm{~A}$ and see online supplementary figure S6). As the patient harbouring XIAP W323X had died before this investigation, we were unable to study the expression and function of this XIAP variant in primary cells. Upon ectopic expression in $293 \mathrm{~T}$ cells, XIAP W323X exhibited largely unimpaired expression as well as unaltered coimmunoprecipitation of RIPK2 in line with an intact BIR2 domain (figure 5A). However, despite preserved RIPK2 recruitment, loss of the XIAP RING domain associated with the W323X mutation is expected to affect NOD signalling through impaired RING domain-dependent ubiquitylation of RIPK2 $2{ }^{47}$ We therefore investigated whether expression of XIAP W323X can restore NOD2 signalling in XIAP-deficient HCT116 cells. ${ }^{52}$ In accordance with a critical function of the XIAP RING domain in NOD2 signalling, MDP-induced,
NOD2-dependent IL-8 release was only observed with wildtype, but not W323X XIAP (figure 5B). Expression of XIAP W323X was associated with reduced DNA binding activity of NF- $\mathrm{kB}$ and impaired NF-kB-dependent transcription in response to MDP (figure 5C, D). This suggested that altered IL-8 secretion occurred as a consequence of impaired XIAP- and NOD2dependent NF- $\mathrm{KB}$ activation. As expected, similar defects in NF- $\mathrm{kB}$ activation and IL-8 secretion were observed with XIAP E99X, which is associated with loss of the BIR2, BIR3 and RING domains of XIAP (figure 5B-D). Of note, reconstitution of XIAP-deficient HCT116 cells with wildtype, but not W323X or E99X XIAP also restored basal IL-8 secretion in the absence of MDP stimulation (figure $5 \mathrm{~B}$ ). These findings resembled observations in primary moDCs harbouring XIAP E99X, where lentiviral transduction of wildtype XIAP restored NOD2-induced secretion and also constitutive IL-8 secretion (figure 4F). As such, these results raised the question of whether XIAP is required for basal and NOD2-induced NF- $\mathrm{\kappa B}$ signalling. In line with this concept, NF-kB-dependent transcription in the 
absence of MDP stimulation was reduced upon expression of W323X and E99X compared to WT XIAP (figure 5D).

Defects observed with XIAP W323X extended to NOD1, as

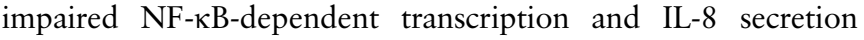
were also observed in response to the NOD1 agonist Tri-DAP (figure 5D-E). Similar observations were made for XIAP E99X (figure 5D-E). HCT116 cells, even in the presence of wildtype XIAP, did not exhibit IL-8 secretion in response to LPS, Pam3CSK4, and TNF $\alpha$ (figure 5F and data not shown). However, IL-8 production in response to phorbol 12-myristate 13-acetate (PMA) and ionomycin (IO) was unimpaired upon expression of W323X and E99X compared to wildtype XIAP suggesting the absence of general defects in IL-8 production or cell viability (figure $5 \mathrm{~F}$ ). In conclusion, these data demonstrate that XIAP E99X and W323X are associated with impaired NOD1/2- and NF- $\mathrm{kB}-$ dependent cytokine responses as a consequence of loss of the XIAP BIR2 and/or RING domain.

\section{Functional analysis of XIAP variants located in the BIR1 and BIR3 domain of XIAP}

XIAP variants previously identified in patients with XLP2 are nonsense and frameshift mutations as well as deletions, which invariably affect the RING domain of XIAP and disrupt NOD1/ 2 signalling. $^{25-30}$ Additionally, XIAP missense mutations were described, which exclusively affect the BIR2 and RING domains and are associated with impaired RIPK2-mediated NOD1/2 signalling. ${ }^{29} 3149$ Thus, disease-associated XIAP variants identified so far share the principle that they affect NOD1/2 signalling through direct or indirect interference with the function of the BIR2 or RING domain. By contrast, mutations G39C and K297T described here are the first disease-associated XIAP missense variants, which are located in the BIR1 and BIR3 domains of XIAP, respectively. We therefore first investigated whether these XIAP variants are associated with impaired expression as commonly observed for XIAP missense variants. ${ }^{29}$ However, XIAP G39C and K297T exhibited expression levels similar to wildtype XIAP upon ectopic expression in $293 \mathrm{~T}$ cells (figure 5A). Moreover, in accordance with an intact BIR2 domain, recruitment of RIPK2 by XIAP G39C and K297T was unimpaired (figure $5 \mathrm{~A}$ ). We then investigated whether reconstitution of XIAP-deficient HCT116 cells with G39C and K297T XIAP restores NOD1/2 signalling. Expression of XIAP K297T was associated with reduced MDP-induced DNA binding activity of $\mathrm{NF}-\kappa \mathrm{B}$ (figure 5C) as well as a modest reduction in MDP-induced and Tri-DAP-induced NF- $\mathrm{BB}$-dependent transcription (figure 5D) and IL-8 secretion (figure 5B and E). By contrast, MDP-induced DNA binding activity of NF- $\mathrm{BB}$ (figure $5 \mathrm{C}$ ) as well as MDP-induced and Tri-DAP-induced IL-8 secretion (figure 5B and E) were unaffected in the presence of XIAP G39C, while a minor reduction in MDP-induced and Tri-DAP-induced NF- $\kappa$ B-dependent transcription was observed (figure 5D). Thus, XIAP K297T is associated with a modest defect in NOD1/2 signalling, while NOD function is largely preserved in the presence of XIAP G39C.

\section{DISCUSSION}

Our studies provide the first systematic analysis of XIAP variants in patients with IBD and reveal their unexpected, frequent occurrence in male patients with paediatric-onset CD. Mutations in XIAP are associated with XLP2, a PID characterised by susceptibility to Epstein Barr virus (EBV) infection and HLH. ${ }^{25}{ }^{45}$ Chronic intestinal inflammation resembling CD has been observed in XLP2. ${ }^{25}{ }^{27-30}$ Moreover, relatives of XLP2 patients with shared XIAP variants occasionally developed intestinal inflammation in the absence of HLH suggesting that XIAP may regulate susceptibility to IBD independent of lymphoproliferative disease. ${ }^{27-29}$ In accordance with this concept, Worthey et al described a patient with early onset CD in the absence of lymphoproliferative disease, who carried a missense mutation in XIAP. ${ }^{31}$ Moreover, a recent summary of patients with XIAP mutations highlighted the variability of clinical manifestations and revealed several cases with $\mathrm{CD}$ as the sole clinical manifestation. ${ }^{30}$ Our identification of XIAP E99X in a patient with early onset CD provided the rationale for the first systematic screening for XIAP variants in IBD. These analyses revealed the frequent occurrence of private variants in XIAP, which were restricted to male patients with paediatric-onset CD (age $\leq 16$ years according to group A1 of the Montreal classification, ${ }^{51}$ and $\mathrm{A} 1 \mathrm{a} / \mathrm{b}$ of the Paris classification ${ }^{12}$ ). Four novel and private XIAP variants were found among 96 male patients with paediatric-onset CD, together reflecting about $4 \%$ of patients in this subset of CD patients. CD patients carrying those variants showed small and large intestinal involvement, perianal disease and stricturing behaviour, while the location of small intestinal disease and the presence of penetrating behaviour differed among patients. Together, these results reveal a common association of XIAP variants with male, paediatric-onset CD independent of the occurrence of lymphoproliferative disease and warrant future systematic sequencing of XIAP in this subset of CD patients. While we did not detect genetic variants in XIAP in a large cohort of adult-onset CD, we observed considerable variation in the age of onset of intestinal inflammation in paediatric patients carrying such variants. Given these findings as well as the previous description of two patients with adult-onset IBD and mutations in XIAP, ${ }^{29} 30$ we suggest that screening for XIAP variants in male patients with severe CD should not be limited to cases of early onset $\mathrm{CD}$, in accordance with previous suggestions. ${ }^{53}$

The clinical course of disease in CD patients with XIAP mutations is often severe and refractory to treatment with immunomodulators and biologicals, which is reflected in the clinical history of patients reported by us and others. ${ }^{30} 31$ For these patients, allogeneic haematopoietic stem cell transplantation (HSCT) may offer a causal therapeutic approach able to restore XIAP expression and function at least in bone marrow-derived cells. HSCT has thereby demonstrated the potential to provide lasting remission of intestinal inflammation in CD patients carrying XIAP mutations. ${ }^{26} 3031$ However, considerable morbidity and mortality associated with HSCT in patients with XIAP mutations $^{26}$ suggests that such treatment should be limited to selected individuals with severe CD refractory to currently available medical treatment.

The association of XIAP variants with IBD and PID is reminiscent of a number of PIDs that manifest as IBD and supports the concept of immunodeficiency underlying a subset of IBD cases. ${ }^{8-11}{ }^{19-24}$ It is noteworthy in this context that the majority of XIAP variants identified here were associated with defects in NOD2, a microbial pattern recognition receptor and CD susceptibility locus. ${ }^{54} 55$ Immune defects in patients with XIAP mutations may thus extend beyond the known susceptibility to EBV and may contribute to intestinal inflammation. This is supported by the observation that Xiap-deficient mice exhibit impaired NOD2 function and susceptibility to bacterial infections in a manner resembling that observed for Nod2-deficient mice. ${ }^{465657}$ Recent findings of a critical role of NOD2 in viral recognition $^{58} 59$ and the crosstalk between viruses and bacteria $^{60} 61$ thereby suggest that NOD2-dependent immunodeficiency in patients with XIAP variants may extend to 
enteropathogenic viruses with potential implications for the pathogenesis of $\mathrm{CD}$. However, despite a clear association between NOD2 variants and IBD, particularly in patients homozygous for NOD2 risk alleles, ${ }^{54}$ it remains to be investigated whether intestinal inflammation in patients harbouring XIAP mutations is indeed related to NOD dysfunction.

Finally, several observations suggest that the loss of functional XIAP is not sufficient to elicit intestinal inflammation but rather requires the presence of other genetic or environmental factors: As such, intestinal inflammation exhibits incomplete penetrance in individuals with shared XIAP variants, and neither the location nor type of mutation predicts the clinical manifestations associated with XIAP mutations. ${ }^{29} 30$ This contrasts with findings made for homozygous loss-of-function mutations in IL10, IL10RA and IL10RB, which were invariably associated with intestinal inflammation manifesting within the first months of life as well as the absence of homozygosity of these mutations in healthy relatives. ${ }^{9-11} 1314$ Consistent with these observations in humans, deficiency in Il10 and Il10rb but not in Xiap is associated with spontaneous intestinal inflammation in mice. ${ }^{15} 16$ While the association of XIAP variants with NOD1/2 defects and susceptibility to infection are in line with the potential involvement of a microbial trigger in the aetiology of intestinal inflammation, studies by Rigaud et al demonstrated that not only environmental but also genetic factors may influence the clinical presentation of genetic variants in XIAP. ${ }^{62}$

In conclusion, our studies reveal an association between XIAP variants and male, paediatric-onset $\mathrm{CD}$. The known association between XIAP mutations and PID thereby supports the notion that a subset of IBD cases may result from immunodeficiency. Moreover, these findings may provide a mechanistic basis for the unexplained observation of functional NOD2 defects in the absence of genetic NOD2 variants. ${ }^{63}$

\footnotetext{
Author affiliations

${ }^{1}$ Department of Internal Medicine I, University Medical Center Schleswig-Holstein, Kiel, Germany

${ }^{2}$ Department of General Pediatrics, University Medical Center Schleswig-Holstein, Kiel, Germany

${ }^{3}$ Institute of Clinical Molecular Biology, Christian-Albrechts-University of Kiel, Kiel, Germany

${ }^{4}$ Sanford-Burnham Medical Research Institute, La Jolla, California, USA

${ }^{5}$ Clinical Bioinformatics, Saarland University, Saarbrücken, Germany

${ }^{6}$ Children's Hospital, Medical Faculty Carl Gustav Carus, Technische Universität

Dresden, Germany

${ }^{7}$ Department of General Pediatrics, University Medical Center Schleswig-Holstein, Lübeck, Germany

${ }^{8}$ Institute of Pathology, University Medical Center Schleswig-Holstein, Lübeck, Germany

${ }^{9}$ Institute of Pathology, University Medical Center Schleswig-Holstein, Kiel, Germany
}

Acknowledgements We would like to acknowledge the help and support of the patients and their families who contributed to this study. This work was supported by the Deutsche Forschungsgemeinschaft (DFG) (ZE 814/6-1) and the European Research Council (ERC Starting Grant agreement number 336528) (SZ) as well as an intramural research grant by the Medical Faculty of the CAU (to YZ). PBS57-loaded and unloaded mouse CD1d tetramers were obtained through the NIH Tetramer Facility. Exome sequencing was partially supported by Siemens Healthcare. Infrastructure support was received from the PopGen biobank and the DFG Cluster of Excellence 306 'Inflammation at Interfaces'. We thank B Vogelstein (Johns Hopkins University) for the gift of XIAP ${ }^{-l y}$ HCT116 cells.

Contributors $Y Z, S M, E B, K P, J H, S B$ performed functional studies. BSP, AK and AF performed genetic analyses. GM analysed XIAP protein structure. MK, HB, MS, $M L, C R$ and ACF contributed to the clinical and histopathological characterisation of the patients. PR, JCR and SS contributed to the coordination of studies. SZ and AF designed the study, coordinated the experimental work and wrote the manuscript with input from coauthors. All authors discussed the results and commented on the manuscript.
Funding Deutsche Forschungsgemeinschaft (DFG).

Competing interests None.

Ethics approval The ethics committees of the Christian-Albrechts-University Kiel and the Technical University Dresden.

Provenance and peer review Not commissioned; externally peer reviewed.

\section{REFERENCES}

1 Molodecky NA, Soon IS, Rabi DM, et al. Increasing incidence and prevalence of the inflammatory bowel diseases with time, based on systematic review. Gastroenterology 2012;142:46-54. e42; quiz e30.

2 Khor B, Gardet A, Xavier RJ. Genetics and pathogenesis of inflammatory bowel disease. Nature 2011:474:307-17.

3 Kaser A, Zeissig S, Blumberg RS. Inflammatory bowel disease. Annu Rev Immunol 2010;28:573-621.

4 Abraham C, Cho JH. Inflammatory bowel disease. N Eng/ J Med 2009;361:2066-78.

5 Jostins L, Ripke S, Weersma RK, et al. Host-microbe interactions have shaped the genetic architecture of inflammatory bowel disease. Nature 2012;491:119-24.

6 Manolio TA, Collins FS, Cox NJ, et al. Finding the missing heritability of complex diseases. Nature 2009;461:747-53.

7 Ruemmele FM, El Khoury MG, Talbotec C, et al. Characteristics of inflammatory bowel disease with onset during the first year of life. J Pediatr Gastroenterol Nutr 2006:43:603-9.

8 Glocker $E$, Grimbacher B. Inflammatory bowel disease: is it a primary immunodeficiency? Cell Mol Life Sci 2012;69:41-8.

9 Glocker EO, Frede N, Perro M, et al. Infant colitis-it's in the genes. Lancet 2010;376:1272.

10 Glocker EO, Kotlarz D, Boztug K, et al. Inflammatory bowel disease and mutations affecting the interleukin-10 receptor. N Engl J Med 2009;361:2033-45.

11 Kotlarz D, Beier R, Murugan D, et al. Loss of interleukin-10 signaling and infantile inflammatory bowel disease: implications for diagnosis and therapy. Gastroenterology 2012:143:347-55.

12 Levine A, Griffiths A, Markowitz J, et al. Pediatric modification of the Montreal classification for inflammatory bowel disease: the Paris classification. Inflamm Bowel Dis 2011;17:1314-21.

13 Moran CJ, Walters TD, Guo CH, et al. IL-10R polymorphisms are associated with very-early-onset ulcerative colitis. Inflamm Bowel Dis 2013;19:115-23.

14 Begue B, Verdier J, Rieux-Laucat F, et al. Defective IL10 signaling defining a subgroup of patients with inflammatory bowel disease. Am J Gastroenterol 2011;106:1544-55.

15 Kuhn R, Lohler J, Rennick D, et al. Interleukin-10-deficient mice develop chronic enterocolitis. Cell 1993;75:263-74.

16 Spencer SD, Di Marco F, Hooley J, et al. The orphan receptor CRF2-4 is an essential subunit of the interleukin 10 receptor. J Exp Med 1998;187:571-8.

17 Chalaris A, Adam N, Sina C, et al. Critical role of the disintegrin metalloprotease ADAM17 for intestinal inflammation and regeneration in mice. J Exp Med 2010:207:1617-24.

18 Blaydon DC, Biancheri P, Di WL, et al. Inflammatory skin and bowel disease linked to ADAM17 deletion. N Engl J Med 2011;365:1502-8.

19 Werlin SL, Chusid MJ, Caya J, et al. Colitis in chronic granulomatous disease. Gastroenterology 1982;82:328-31.

20 Dupuis-Girod S, Medioni J, Haddad E, et al. Autoimmunity in Wiskott-Aldrich syndrome: risk factors, clinical features, and outcome in a single-center cohort of 55 patients. Pediatrics 2003;111:e622-7.

21 Marks DJ, Harbord MW, MacAllister R, et al. Defective acute inflammation in Crohn's disease: a clinical investigation. Lancet 2006;367:668-78.

22 Segal AW, Loewi G. Neutrophil dysfunction in Crohn's disease. Lancet 1976;2:219-21.

23 Casanova JL, Abel L. Revisiting Crohn's disease as a primary immunodeficiency of macrophages. J Exp Med 2009;206:1839-43.

24 Marks DJ, Rahman FZ, Sewell GW, et al. Crohn's disease: an immune deficiency state. Clin Rev Allergy Immunol 2010;38:20-31.

25 Rigaud S, Fondaneche MC, Lambert N, et al. XIAP deficiency in humans causes an X-linked lymphoproliferative syndrome. Nature 2006:444:110-14.

26 Marsh RA, Rao K, Satwani P, et al. Allogeneic hematopoietic cell transplantation for XIAP deficiency: an international survey reveals poor outcomes. Blood 2013;121:877-83.

27 Yang $X$, Kanegane $\mathrm{H}$, Nishida $\mathrm{N}$, et al. Clinical and genetic characteristics of XIAP deficiency in Japan. J Clin Immunol 2012;32:411-20.

28 Filipovich $\mathrm{AH}$, Zhang $\mathrm{K}$, Snow $\mathrm{AL}$, et al. X-linked lymphoproliferative syndromes: brothers or distant cousins? Blood 2010;116:3398-408.

29 Pachlopnik Schmid J, Canioni D, Moshous D, et al. Clinical similarities and differences of patients with X-linked lymphoproliferative syndrome type 1 (XLP-1/ SAP deficiency) versus type 2 (XLP-2/XIAP deficiency). Blood 2011;117:1522-9.

30 Speckmann C, Lehmberg K, Albert MH, et al. X-linked inhibitor of apoptosis (XIAP) deficiency: The spectrum of presenting manifestations beyond hemophagocytic lymphohistiocytosis. Clin Immunol 2013;149:133-41. 
31 Worthey EA, Mayer AN, Syverson GD, et al. Making a definitive diagnosis: successful clinical application of whole exome sequencing in a child with intractable inflammatory bowel disease. Genet Med 2011;13:255-62.

32 Li H, Durbin R. Fast and accurate short read alignment with Burrows-Wheeler transform. Bioinformatics 2009;25:1754-60.

33 Li H, Handsaker B, Wysoker $\mathrm{A}$, et al. The sequence alignment/map format and SAMtools. Bioinformatics 2009;25:2078-9.

34 McKenna A, Hanna M, Banks E, et al. The Genome Analysis Toolkit: a MapReduce framework for analyzing next-generation DNA sequencing data. Genome Res 2010;20:1297-303.

35 Wang K, Li M, Hakonarson H. ANNOVAR: functional annotation of genetic variants from high-throughput sequencing data. Nucleic Acids Res 2010;38:e164.

36 Koboldt DC, Zhang Q, Larson DE, et al. VarScan 2: somatic mutation and copy number alteration discovery in cancer by exome sequencing. Genome Res 2012:22:568-76.

37 Weckx S, Del-Favero J, Rademakers $\mathrm{R}$, et al. novoSNP, a novel computational too for sequence variation discovery. Genome Res 2005:15:436-42.

38 Zeissig S, Dougan SK, Barral DC, et al. Primary deficiency of microsomal triglyceride transfer protein in human abetalipoproteinemia is associated with loss of CD1 function. J Clin Invest 2010;120:2889-99.

39 Zeissig S, Fromm A, Mankertz J, et al. Butyrate induces intestinal sodium absorption via Sp3-mediated transcriptional up-regulation of epithelial sodium channels. Gastroenterology 2007;132:236-48.

40 Krieg A, Correa RG, Garrison JB, et al. XIAP mediates NOD signaling via interaction with RIP2. Proc Natl Acad Sci U S A 2009;106:14524-9.

41 Hong JC, Ivanov NV, Hodor $P$, et al. Identification of new human cadherin genes using a combination of protein motif search and gene finding methods. J Mol Biol 2004:337:307-17.

42 Gloerich M, Bos JL. Epac: defining a new mechanism for cAMP action. Annu Rev Pharmacol Toxicol 2010;50:355-75.

43 Kawasaki H, Springett GM, Mochizuki N, et al. A family of CAMP-binding proteins that directly activate Rap1. Science 1998;282:2275-9.

44 Saule $P$, Trauet J, Dutriez $V$, et al. Accumulation of memory $T$ cells from childhood to old age: central and effector memory cells in CD4(+) versus effector memory and terminally differentiated memory cells in CD8(+) compartment. Mech Ageing Dev 2006;127:274-81.

45 Marsh RA, Madden L, Kitchen BJ, et al. XIAP deficiency: a unique primary immunodeficiency best classified as X-linked familial hemophagocytic lymphohistiocytosis and not as X-linked lymphoproliferative disease. Blood 2010;116:1079-82

46 Bauler LD, Duckett CS, O'Riordan MX. XIAP regulates cytosol-specific innate immunity to Listeria infection. PLoS Pathog 2008;4:e1000142.

47 Damgaard RB, Nachbur U, Yabal M, et al. The ubiquitin ligase XIAP recruits LUBAC for NOD2 signaling in inflammation and innate immunity. Mol Cell 2012;46:746-58.

48 Lipinski S, Grabe N, Jacobs G, et al. RNAi screening identifies mediators of NOD2 signaling: implications for spatial specificity of MDP recognition. Proc Natl Acad Sci U S A 2012;109:21426-31.
49 Damgaard RB, Fiil BK, Speckmann C, et al. Disease-causing mutations in the XIAP BIR2 domain impair NOD2-dependent immune signalling. EMBO Mol Med 2013;5:1278-95

50 Vince JE, Wong WW, Gentle I, et al. Inhibitor of apoptosis proteins limit RIP3 kinase-dependent interleukin-1 activation. Immunity 2012;36:215-27.

51 Satsangi J, Silverberg MS, Vermeire $S$, et al. The Montreal classification of inflammatory bowel disease: controversies, consensus, and implications. Gut 2006;55:749-53.

52 Cummins JM, Kohli M, Rago C, et al. X-linked inhibitor of apoptosis protein (XIAP) is a nonredundant modulator of tumor necrosis factor-related apoptosis-inducing ligand (TRAIL)-mediated apoptosis in human cancer cells. Cancer Res 2004;64:3006-8.

53 Speckmann C, Ehl S. XIAP deficiency is a mendelian cause of late-onset IBD. Gut 2014:63:1031-2

54 Hugot JP, Chamaillard M, Zouali H, et al. Association of NOD2 leucine-rich repeat variants with susceptibility to Crohn's disease. Nature 2001:411:599-603.

55 Hampe J, Cuthbert A, Croucher PJ, et al. Association between insertion mutation in NOD2 gene and Crohn's disease in German and British populations. Lancet 2001;357:1925-8

56 Kobayashi KS, Chamaillard M, Ogura Y, et al. Nod2-dependent regulation of innate and adaptive immunity in the intestinal tract. Science 2005;307:731-4.

57 Prakash $\mathrm{H}$, Albrecht M, Becker D, et al. Deficiency of XIAP leads to sensitization for Chlamydophila pneumoniae pulmonary infection and dysregulation of innate immune response in mice. J Biol Chem 2010;285:20291-302.

58 Lupfer C, Thomas PG, Anand PK, et al. Receptor interacting protein kinase 2-mediated mitophagy regulates inflammasome activation during virus infection. Nat Immunol 2013;14:480-8.

59 Sabbah A, Chang TH, Harnack R, et al. Activation of innate immune antiviral responses by Nod2. Nat Immunol 2009;10:1073-80.

$60 \mathrm{Kim}$ YG, Park JH, Reimer T, et al. Viral infection augments Nod1/2 signaling to potentiate lethality associated with secondary bacterial infections. Cell Host Microbe 2011;9:496-507.

61 Coulombe F, Fiola S, Akira S, et al. Muramyl dipeptide induces NOD2-dependent Ly6C(high) monocyte recruitment to the lungs and protects against influenza virus infection. PLoS One 2012:7:e36734.

62 Rigaud S, Lopez-Granados E, Siberil S, et al. Human X-linked variable immunodeficiency caused by a hypomorphic mutation in XIAP in association with a rare polymorphism in CD40LG. Blood 2011;118:252-61.

63 Seidelin JB, Broom OJ, Olsen J, et al. Evidence for impaired CARD15 signalling in Crohn's disease without disease linked variants. PLoS One 2009;4:e7794.

64 Rufer N, Zippelius A, Batard P, et al. Ex vivo characterization of human CD8+ T subsets with distinct replicative history and partial effector functions. Blood 2003:102:1779-87.

65 Geginat J, Lanzavecchia A, Sallusto F. Proliferation and differentiation potential of human CD8+ memory T-cell subsets in response to antigen or homeostatic cytokines. Blood 2003;101:4260-6.

66 Amyes E, McMichael AJ, Callan MF. Human CD4+ T cells are predominantly distributed among six phenotypically and functionally distinct subsets. I Immunol 2005;175:5765-73. 